\section{Zur Diagnose und Prognose der kindlichen Lungentuberkulose.)} Von A. Czerny.

M. H.! Bei der Diagnose der Lungentuberkulose der Erwachsenen steht die Spitzentuberkulose im Vordergrunde des Interesses, bei der der Kinder die Bronchialdrïsentuberkulose. Nach dem gegenwärtigen Stande unseres Wissens gibt es zwar keine primäre und isolierte Bronchialdrüsentuberkulose. Diese ist vielmehr, abgesehen von den wenigen Fällen einer vom $A$ bdomen auf dem Lymphwege aufsteigenden Tuberkuloseinfektion, stets die Folge eines primären Lungeninfektes. Ein kleinster, stecknadelkopf- bis erbsengroßer Tuberkelherd, der selbst bei der Obduktion nur bei genauester und besonders darauf gerichteter Untersuchung zu finden ist, kann zu einer hochgradigen Infektion der Bronchialdrüsen Veranlassung geben. Dieses Mißverhältnis zwischen dem primären Herd und der sekundären Bronchialdrüsenaffektion bringt es mit sich, daß wir vielfach den ersteren klinisch nicht nachweisen und nur aus dem Befunde einer Bronchialdrüsentuberkulose erschließen können. Eben deshalb ist aber die Diagnose der Bronchialdrüsentuberkulose für das Kindesalter besonders wichtig. Die Untersuchung mit Röntgenstrahlen hat die älteren klinischen Methoden stark zurückgedrängt. Es darf aber nicht verschwiegen werden, daß auch sie nur Unvollkommenes leistet. Leicht nachweisbar sind mit Röntgenstrahlen hochgradige Bronchialdrüsenerkrankungen, bei welchen die tuberkulösen Drüsen zahlreich vorhanden und zu ansehnlichen Tumoren angewachsen sind. In den Fällen, in welchen nur eine oder die andere Drüse tuberkulös ist und als Tumor nicht den HerzgefäBschatten überragt, läßt uns auch das Röntgenbild im Stich.

Ein erschwerender und bemerkenswerter Umstand für die Diagnose der beginnenden Lungen- and Bronchialdrüsentuberkulose liegt darin, daß stärkere Schwankungen der Körpertemperatur noch weniger als bei Erwachsenen auf Tuberkulose schließen lassen. Wir wissen, daß Temperaturschwankungen bis $38^{\circ}$ für viele sensible Kinder charakteristisch sind, an denen sich auch bei jahrelanger Beobachtung nicht der geringste Beweis für eine Tuberkuloseinfektion erbringen läßt. Nur Temperaturschwankungen, die $38^{\circ}$ (bei rektaler Messung) erreichen oder übersteigen, rechtfertigen den Verdacht einer Tuberkulose beim Kinde. Ebenso erscheint es mir notwendig, darauf hinzuweisen, daß der asthenische Habitus nichts mit Tuberkulose $\mathrm{zu}$ tun hat. Immer wieder werden uns Kinder zur Untersuchung auf Tuberkulose zugeführt, die diesen Habitus aufweisen, und nur selten gelingt dabei der Nachweis einer Tuberkuloseinfektion. Dagegen finden wir diese relativ oft bei Kindern, deren guter Habitus es zweifellos verschuldet, daß die Tuberkulose erst spät erkannt wird.

Aus dem positiven Ergebnis einer kutanen Tuberkulinreaktion allein auf den Bestand ejner Bronchialdrüsentuberkulose zu schließen, ist nicht zulässig. Wenn auch der statistische Nachweis die Tatsache bestätigt, daß in der Mehrzahl aller Fälle die Tuberkuloseinfektion die Lunge und die Bronchialdrüsen betrifft, so darf doch nicht vergessen werden, daß die Tuberkulose noch auf höchst mannigfaltigen anderen Wegen in den Organismus des Kindes eindringen kann.

Wichtig erscheint es mir, hervorzuheben, daß die Bronchialdrüsentuberkulose in beginnenden und leichteren Fällen stets nur eine Wahrscheinlichkeitsdiagnose bleibt. Dies verlangt Beachtung bei der Wertung von Heilstatistiken.

Was die Diagnostik der Lungentuberkulose anbelangt, so

1) Ergänzungsreferat zu den Vorträgen von Goldscheider u. Kraus, auf Ersuchen des Vorstandes d's Vereins für Innere Medizin u. Kindhik, erstattet am 10. XII. 1917. möchte ich zuerst die Miliartuberkulose der Lunge erwähnen. Seitdem es möglich ist, von der Lunge mit Röntgenstrahlen Momentaufnahmen zu machen, ist die Diagnose relativ frühzeitig und mit Sicherheit zu stellen. Wir erkennen auf diese Weise die Miliartuberkulose der Lunge schon in einem Stadium, in dem unsere übrigen Untersuchungsmethoden noch versagen. Daß eine sichergestellte Krankheit dieser Art heilen kann, glaube ich nicht, doch möchte ich darauf hinweisen, daß auch ein recht hochgradiger Befund im Rötngenbild nicht unbedingt einen rapiden schləchten Verlauf voraussehen läßt. Ich habe Kinder gesehen, die sich mit einer Miliartuberkulose der Lunge monatelang in einem guten Zustand hielten. Nicht unerwähnt darf dabei bl iben, daß es Bronchopneumonien gibt, welche ein der Miliartuberkulose sehr ähnliches Röntgenbild zeigen.

Von der Lungentuberkulose der Kinder muß an erster Stelle angeführt werden, daß wir sie nicht, wie bei Erwachsenen, in den Iungenspitzen, sondern in der Hilusgegend $\mathrm{zu}$ suchen haben. Wohl kommt auch hier und da eine andere Lokalisation vor, doch prävaliert die in der Hilusgegend so stark, daß wir diese in jedem suspekten Falle besonders gründlich untersuchen müssen. Auch das vorzeitige Absinken des Thorax ändert nichts an dieser Tatsache. Es begünstigt keinesfalls bei Kindern die Entstehung einer Spitzentuberkulose.

Die Sputumuntersuchung auf Bazillen ergibt bei der Lungentuberkulose der Kinder nur selten ein positives Resultat. Die Erkennung beginnender Erkrankungen gestattet sie nicht. Wo sich Bazillen finden, besteht bereits ein weit vorge shrittener Prozeß. Dies erklärt sich daraus, daß Lungentuberkulose bei Kindern oft als Miliartuberkulose und ebenso häufig bis zum Ende als geschlossene Tuberkulose verläuft. Offene Tuberkulosen kommen aber, wenn auch relativ selten, in jedem Kindesalter zur Beobachtung. Sind Bazillen einmal nachgewiesen, so verschwinden sie niemals bis zum Exitus aus dem Sputum. Mir ist wenigstens kein Fall bekannt, in dem eine offene Tubërkulose bei einem Kinde erloschen und die Bazillen aus dem Sputum verschwunden wären.

Stets ist mir bei der Lungentuberkulose der Gegensatz zwischen der Privatpraxis und der klinischen und poliklinischen Erfahrung aufgefallen. In den besser situierten Gesellschafts. schichten bekommt man hier und da einmal eine Drüsen- oder Knochentuberkulose oder auch eine Peritonitis und Meningitis tuberculosa $\mathrm{zu}$ sehen. Lungentuberkulose ist bei den Kindern dieser Kreise bis zum Pubertätsalter eine Seltenheit. In der Klinik und Poliklinik sehen wir dagegen Lungentuberkulose recht oft. Ich möchte daraus den Schluß ziehen, daß sich die Lungentuberkulose bei Kindern nur dort findet, wo der wiederholten und massigen Infektion Tür und Tor geöffnet ist.

Die geschlossene Form der Lungentuberkulose gelangt bei Kindern in zwei Formen zur Beobachtung, entweder so gering. fügig, daß wir sie nur mit einer gewissen Wahrscheinlichkeit diagnostizieren können, oder in Form eines mehr oder minder großen, leicht nachweisbaren Lungeninfiltrates. $E_{\mathbb{S}}$ ist merkwürdig, daß wir stets nur diese beiden Extreme sehen und nicht Gelegenheit finden, die Entstehung der großen Lungen. infiltrate von ihren Anfängen an zu verfolgen. Man könnte daraus folgern, daß die Entwicklung der Lungentuberkulose bei Kindern unter so geringfügigen Symptomen erfolgt, daß sie jedesmal übersehen werden. Dies ist jedoch unwahrscheinlich. Ich glaube vielmehr, daß es sich von vornherein um Infekte verschiedenen Grades handelt. Die kleinen Infekte haben minimale Lungenherde zur Folge. Diese können, wie die klinische und die pathologisch-anatomische Erfahrung lehrt, ausheilen. Die großen Infekte führen akut zur Entstehung großer Lungenherde, welche nur unter einer besonderen Bedingung zum Stillstand und vielleicht zur Heilung zu bringen sind. Diese Bedingung ist die Möglichkeit der Ausschaltung der kranken Lunge durch künstlichen Pneumothorax. Glücklicherweise betrifft die Tuberkulose bei Kindern häufig vorwiegend eine Lunge. Dies begünstigt die Anwendung der $\mathrm{Me}$ thode der Pneumothoraxbehandlung. Leider setzen manchmal schon frühzeitig Verwachsungen der Pleurablätter dieser Therapie Schranken. Trotzdem möchte ich zu behaupten wagen, daß bisher die Pneumothoraxbehandlung die einzige ist, welche bei dem Befunde eines größeren, sicher nachweisbaren 
Lungenherdes beim Kinde Rettung verspricht. Wohl sieht man einzelne Kinder, bei denen die Lungentuberkulose ohne diese Behandlung langsam verläuft. Es gelingt, die Kinder unter günstigen Behandlungsbedingungen längere Zeit bei gutem Aussehen und im guten Allgemeinzustand zu erhalten. Bei genauer Untersuchung ergibt sich aber, daß die Lungenherde trotzdem, wenn auch langsam, fortschreiten und schonungslos zum Tode führen. Ob durch die Pneumothoraxbehandlung Heilungen von Kindern zu erzielen sind, die ein langes Leben hindurch anhalten, bleibt noch abzuwarten. Soviel können wir jedoch bereits sagen, daß Kinder mit Pneumothoraxbehandlung jahrelang leben und gut leben, die ohne diese Behandlung schon lange begraben wären.

Bei den kleinen Lungeninfekten, welche wir mit den allgemein üblichen Untersuchungsmethoden überhaupt nicht und mit Zuhilfenahme des Röntgenbildes nur mit mehr oder weniger größerer Wahrscheinlichkeit diagnostizieren können, ist die Prognose im Kindesalter günstig, und zwar um so sicherer günstig, je älter das betroffene Kind ist. Es scheint mir aber notwendig zu sein, darauf hinzuweisen, daß die Prognose unter diesen Umständen auch günstig bleibt, wenn wir den Kindern nicht alles bieten, was der Luxus bei der Tuberkulosebehandlung gestattet. Ich erwähne dies mit Absicht, um vor. der Ueberschätzung aller unserer bisherigen Behandlungsmethoden der Lungentuberkulose zu warnen.

Es scheint mir erlaubt, von einer guten Prognose zu sprechen, wenn die Lungentuberkulose der Kinder bis zum Pubertätsalter im latenten Stadium verbleibt. Damit ist noch nicht ausgeschlossen, daß sie im späteren Leben gelegentlich vôn neuem aufflackern und einen ernsteren Verlauf nehmen kann. Mit größter Sicherheit könnten wir von einer guten Prognose sprechen, wenn während des Latenzstadiums die Reaktion auf Tuberkulin, nachdem sie längere Zeit hindurch positiv war, negativ wird. Selter hat dies im Tierexperiment als möglich bewiesen, und Cronquist gibt an, solche Heilungen bei Kindern beobachtet zu haben. Die Tuberkulinreaktion würde unter diesen' Umständen eine ähnliche Bedeutung erlangen, wie $\check{r}^{-}$ von vielen Autoren der Wa.R. bei Lues zugeschrieben wir Darüber müßten uns aber erst zahlreiche weitere Untersuchung Aufklärung bringen. 